\title{
Structural Characterization and Expression Analysis of the SERK/SERL Gene Family in Rice (Oryza sativa)
}

\author{
Bhumica Singla, Jitendra P. Khurana, and Paramjit Khurana \\ Department of Plant Molecular Biology, University of Delhi South Campus, Benito Juarez Road, New Delhi 110021, India \\ Correspondence should be addressed to Paramjit Khurana, paramjitkhurana@hotmail.com
}

Received 7 November 2008; Accepted 17 June 2009

Recommended by Silvana Grandillo

\begin{abstract}
Somatic embryogenesis (SE) is the developmental restructuring of somatic cells towards the embryogenic pathway and forms the basis of cellular totipotency in angiosperms. With the availability of full-length cDNA sequences from Knowledge-based Oryza Molecular Biological Encylopedia (KOME), we identified the leucine-rich repeat receptor-like kinase (LRR-RLK) genes from rice (Oryza sativa), which also encompasses genes involved in regulating somatic embryogenesis. Eight out of eleven of the rice SERK and SERL (SERK-like) genes have the TIGR annotation as (putative) brassinosteroid insensitive 1-associated receptor kinase (precursor). Real-time polymerase chain reaction analysis was undertaken to quantify transcript levels of these 11 genes. Most of these genes were upregulated by brassinosteroids although only a few of these displayed auxin induction. The expression profile of these genes is nearly uniform in the zygotic embryogenic tissue, but the expression pattern is more complex in the somatic embryogenic tissue. It is likely that OsSERKs and OsSERLs may be involved in somatic embryogenesis and also perform a role in morphogenesis and various other plant developmental processes. Functional validation of these somatic embryogenesis receptorlike kinase genes may help in elucidating their precise functions in regulating various facets of plant development.
\end{abstract}

Copyright ( $) 2009$ Bhumica Singla et al. This is an open access article distributed under the Creative Commons Attribution License, which permits unrestricted use, distribution, and reproduction in any medium, provided the original work is properly cited.

\section{Introduction}

The ability to perceive and process information from chemical signals via cell surface receptors is a basic property of all living systems. The putative role of receptor-like kinases (RLKs), which belong to a large gene family [1], in developmental processes is to transduce environmental signals and/or information from the neighboring cells to trigger specific responses. One such developmental process is somatic embryogenesis which depends not only on the cell type employed but also on the culture conditions including composition of the nutrient medium [2].

SERK is a small gene family with at least five members in Arabidopsis, the model dicot plant [3]. Similarly, rice is considered a model plant among monocots [4]. Since the grasses are inferred to have monophyletic origin [5], information obtained from rice is invariably helpful for studying other cereal crops as well. Although earlier workers reported the isolation and characterization of OsSERK cDNA representing the first SERK from rice [6-8], the present study identifies and comprehensively analyzes the entire SERK-like (SERL) gene family from rice (Oryza sativa). This work involved the identification of SERL gene family members from rice, analysis of their structure, duplication, chromosomal distribution, and phylogenetic relationship. The real-time polymerase chain reaction (RT-PCR) analysis demonstrated that SERKandSERL genes are expressed differentially in developing seed, various organs/tissues, under light or dark, and are regulated by auxin/brassinosteroid treatment in rice, indicating that they may perform specific as well as redundant functions in plant development.

\section{Material and Methods}

2.1. Identification of OsSERK Gene Family in Rice. To identify SERK homologs in rice, the Knowledge-Based Oryza Molecular Biological Encylopedia (KOME) (http:// cdna01.dna.affrc.go.jp/cDNA/), the National Centre for Biotechnology Information (NCBI) (http://www.ncbi.nim .nih.gov/BLAST/nr/EST), and The Institute for Genomic 


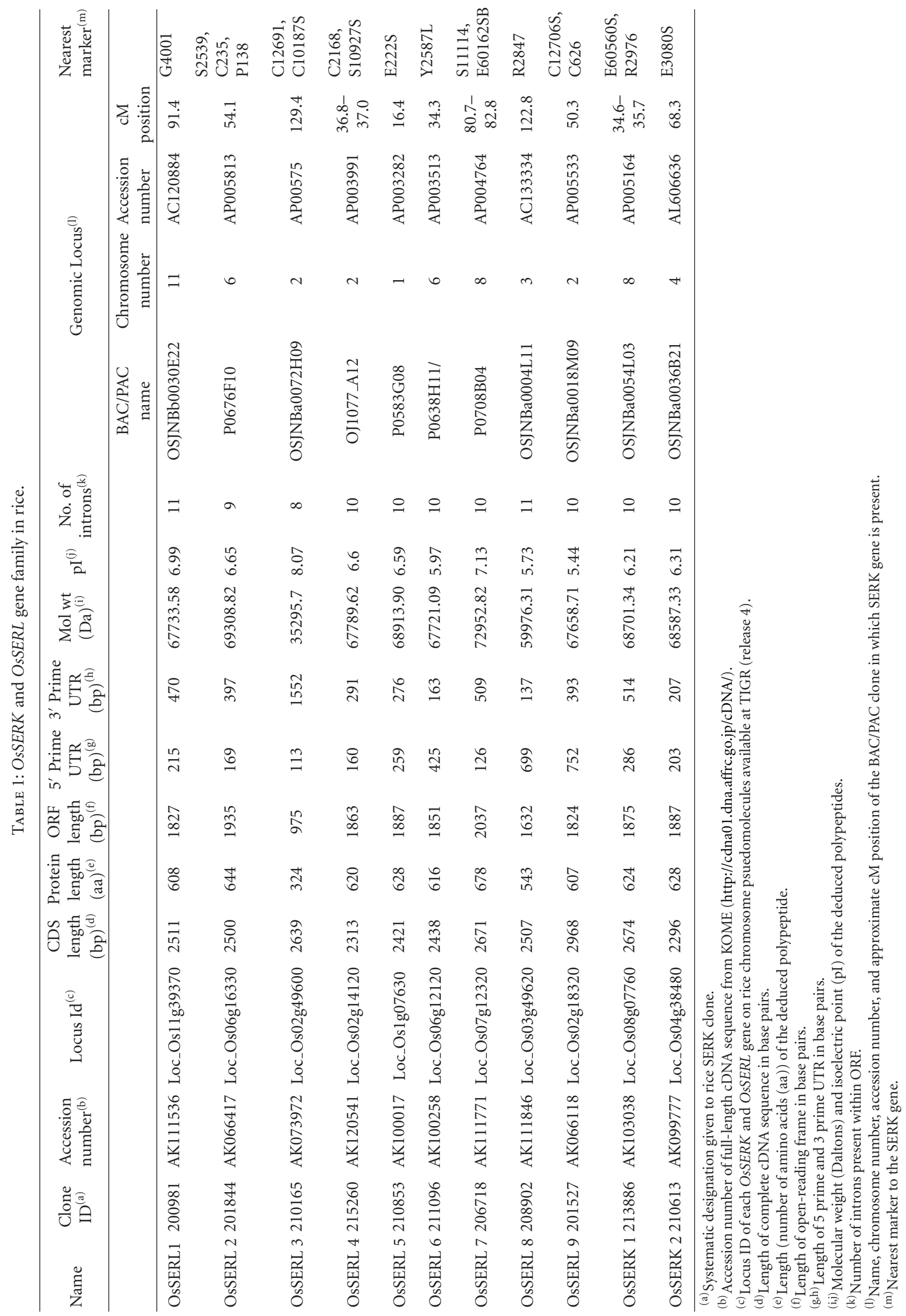



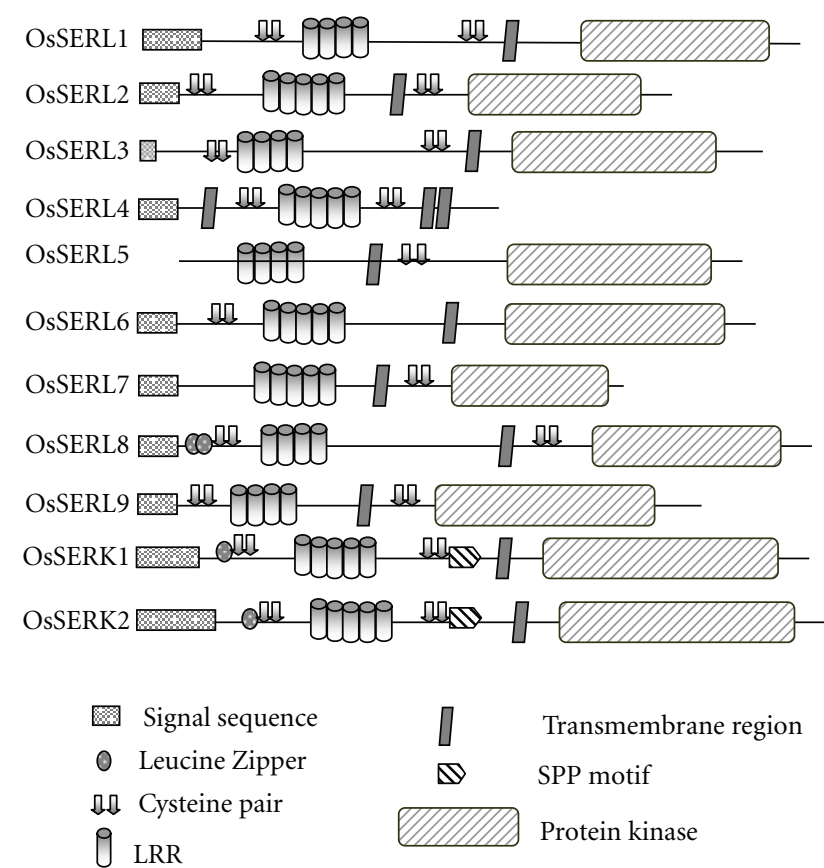

Figure 1: The domains are represented pictorially for the 2 OsSERK and 9 OsSERL proteins. To summarize, a signal peptide sequence is present in all except OsSERL5, one cysteine pair is present in OsSERL5, OsSERL6, and OsSERL7, five LRRs are present in OsSERK1, OsSERK2, OsSERL2, OsSERL4, OsSERL6, and OsSERL7, trans-membrane domain is present in all, SPP motif is present in only two, OsSERK1 and OsSERK2, and a protein kinase domain is present in all except OsSERL4.

Research (TIGR) database (http://www.tigr.org/tdb/edb2/ osa2/osa1/htmls/osa1.html) resources were used. Using the amino acid sequences of the available SERK proteins in TIGR and GenBank, polypeptide records of Viridiplantae, including all land plants and algae with a cut off Evalue of $1 \times 10^{-10}, 55$ sequences (NCBI, 25; TIGR, 30) were retrieved, downloaded, and used to search for their homologs in rice KOME using the TBLASTN program [9] after multiple alignment by ClustalX (version 1.83) program [10]. Additional members of rice SERK gene family were also searched by TBLASTN and BLASTN in the NCBI (nr and est) and TIGR databases, and 11 full-length cDNA sequences were recovered after removing the redundant sequences.

2.2. Sequence Analysis. The pseudomolecules of rice chromosomes available at TIGR (release 4) were used to position OsSERK and OsSERL genes by the BLASTN search. The number and position of exons and introns for individual Oryza sativa somatic embryogenesis receptor kinase (OsSERK) genes were determined by comparison of the cDNAs with their corresponding genomic DNA sequences. The multiple sequence alignment was carried out using ClustalX (version 1.83), and the unrooted phylogenetic tree was generated using neighbour-joining method. Bootstrap values from 1000 replicates are indicated at each node.
The Gene Runner program (version 3.04), and DNASTAR (version 4.0) were used for the DNA and protein analysis.

2.3. Plant Material and Growth Conditions. Rice seeds ( $O$. sativa L. ssp. indica var. Pusa Basmati 1) were soaked overnight in $\mathrm{RO}$ water after sterilization with $0.1 \% \mathrm{HgCl}_{2}$ for 30 minutes. The tissues were harvested from 3/4-dayold seedlings grown on RO-soaked cotton, either in dark or in 14-hour light and 10-hour dark cycle in a culture room maintained at $28 \pm 1^{\circ} \mathrm{C}$. Floral tissue was collected from rice plants grown under field conditions. The callus tissue was raised as described [11]. For auxin treatment, the coleoptiles from 4-day-old etiolated rice seedlings were incubated in KPSC buffer ( $10 \mathrm{mM}$ potassium phosphate, $\mathrm{pH} 6.0,2 \%$ sucrose, $50 \mathrm{mM}$ chloramphenicol) for 16 hours to deplete endogenous auxin; the buffer was changed every hour. The coleoptiles were then transferred to a fresh buffer with $30 \mu \mathrm{M}$ concentration of 2,4-dichlorophenoxy acetic acid (2,4-D) and incubated for 3 hours or with $100 \mathrm{nM}$ epibrassinolide (EBR) for 1 hour [12].

2.4. RNA Isolation and Real-Time PCR. Total RNA was extracted using the RNeasy Plant mini kit (Qiagen, Germany) according to the manufacturer's instructions. The quantitative real-time PCR analysis was performed as described [12]. The primer sequences are listed in Supplementary Table 1(see Table 1 in Supplementary Material available online at doi: 10.1155/2009/539402). The specificity of the reactions was verified by melting curve analysis. The relative mRNA levels for each of the 11 OsSERK and OsSERL genes in RNA isolated from various tissue samples were quantified with respect to the internal standard, actin. At least two independent RNA isolations were used for cDNA synthesis, and each cDNA sample was subjected to real-time PCR analysis in triplicate.

\section{Results and Discussion}

3.1. SERK and SERK-Like (SERL) Gene Family in Rice. The SERK genes are present as small multigene family in Arabidopsis [3], maize [2], sunflower [13], Poa pratensis [14], rice [7], and wheat [15]. Nine nonredundant clones having high sequence similarity with known SERK proteins could be identified by TBLASTN or BLASTN search (International rice genome sequencing project; http://www.tigr.org/tdb/e2kl/osal/; http://www.rgp .dna.affrc.go.jp/). Thus, overall the SERKgene family in rice comprises of two members, OSSERK1 and OsSERK2 [6, 7], and the SERK-like (SERL) gene family comprises of nine members, designated as OsSERL 1 to 9 (Table 1). Among the 11 members, most of them have been annotated as BRI-associated receptor-like kinase, precursor, putative BRIassociated receptor-like kinase and LRR family protein in TIGR.

3.2. Sequence Analysis of OsSERK and OsSERK-Like (OsSERL) Proteins. The deduced molecular mass of rice SERK and SERL peptides ranges from $60 \mathrm{kDa}$ for OsSERL5 


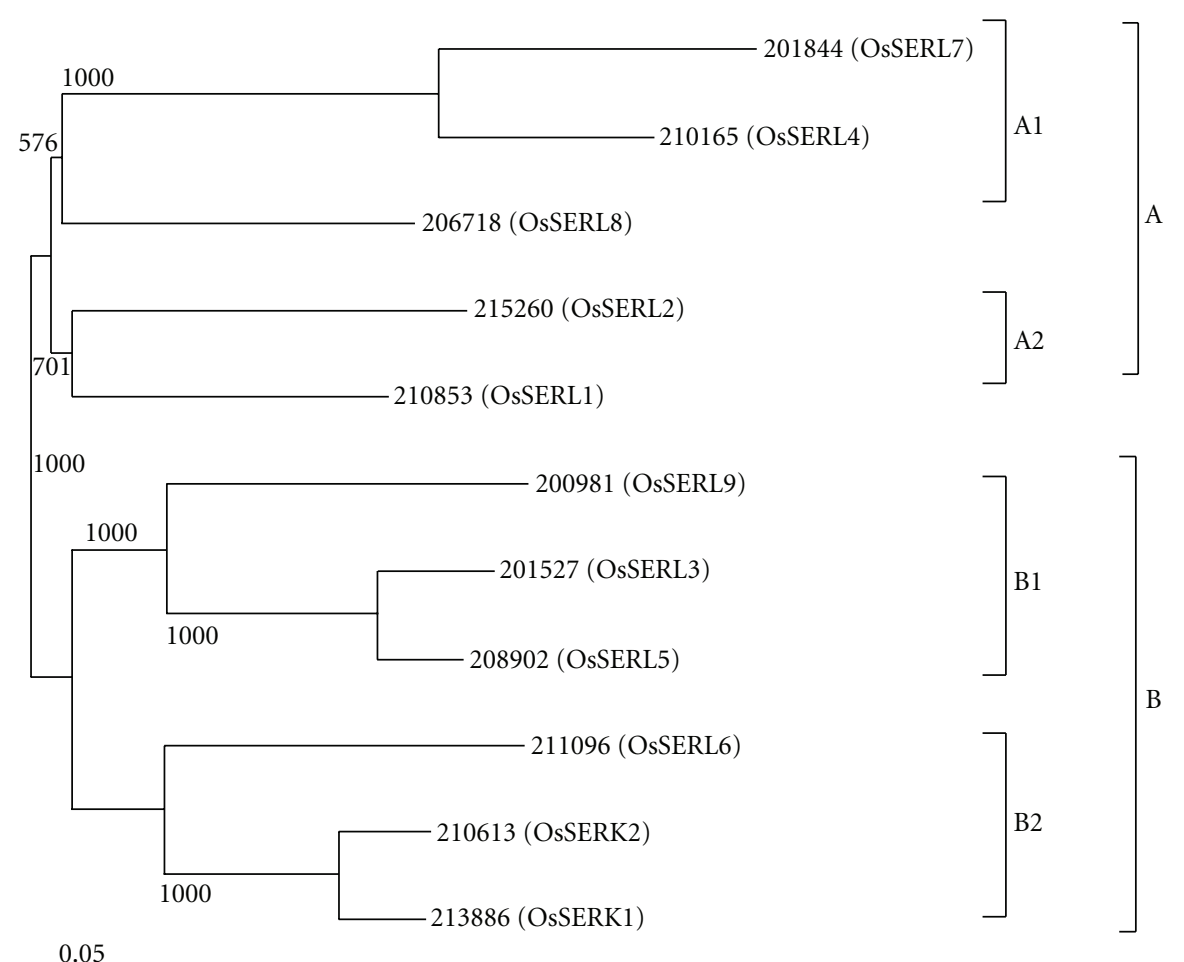

FIGURE 2: Phylogenetic relationship among the rice SERK and SERL proteins. The unrooted tree was generated using ClustalX program by neighbour-joining method. Bootstrap values from 1000 replicates are indicated at each node.

to $73 \mathrm{kDa}$ for OsSERL8, except OsSERL4 which lacks the kinase domain (Table 1). To examine in detail the domain organization of OsSERL proteins, the multiple sequence alignments of the full-length protein sequences were done using the ClustalX program (see Supplementary Figure 1). Among the 11 proteins described, two showed the presence of the SPP motif, and the remaining nine showed the characteristic features of the SERK proteins, except the SPP motif. On the basis of domain search programmes (http://us.expasy.org/prosite/; http://www.ebi.ac.uk/InterProscan/; http://smart.embl/heidelberg.de/; http://www.sanger.ac.uk/software/pfam/index .html), the deduced domains are represented in a pictorial form (Figure 1). To summarize, a signal peptide sequence is present in all except OsSERL5, one cysteine pair is present in OsSERL5, OsSERL6, and OsSERL7, five LRRs are present in OsSERK1, OsSERK2, OsSERL2, OsSERL4, OsSERL6, and OsSERL7, transmembrane domain is present in all, SPP motif is present in only two, OsSERK1 and OsSERK2, and a protein kinase domain is present in all except OsSERL4. Since OsSERL4 and OsSERL7 were showing significant homology $(75 \%)$ at the protein level but OsSERL4 was lacking the kinase domain and also harbouring an unusually long 3'UTR (1552 bp), the sequence of the 3'UTR was aligned with the cDNA region coding for the kinase and C-terminal domain of OsSERL7. It could be clearly deduced from the alignment that OsSERL4 has lost the kinase activity due to changes in certain nucleotide positions and also some changes in the respective frame; even in the absence of the kinase domain the $3^{\prime}$ UTR depicts relic of the original kinase domain. Receptor protein kinases are plasma membrane-bound and play an important role in the perception and transmission of external signals [16, 17]. The protein localization of these genes as shown by the PSORT programme suggests that 9 out of 11 proteins reside in the plasma membrane (OsSERL1, OsSERL2, OsSERL3, OsSERL4, OsSERL6, OsSERL7, OsSERL8, OsSERK1 and OsSERK2), which is the case with most of the SERKs also [18]. On the other hand, OsSERL5 and OsSERL9 are localized to the inner mitochondrial membrane and endoplasmic reticulum, respectively.

3.3. Gene Structure and Phylogenetic Analysis of Rice SERK and SERL Genes. A pairwise analysis of the full-length OsSERK protein sequences indicated that the overall identities among the OsSERK and OsSERL proteins range from $35 \%$ to $87 \%$ and from $25 \%$ to $87 \%$ with the Arabidopsis SERK proteins, respectively. On comparing the full-length cDNA sequences with the corresponding genomic DNA sequence, it appears that the coding sequences of the majority of the OsSERLgenes (7 among 9) have 10-11 introns (Table 1). To examine the phylogenetic relationship among the rice SERK and SERL proteins, an unrooted tree was constructed from alignments of the full-length SERK protein sequences (Figure 2). The sequences formed separate clusters and were grouped into two major groups (groups $A$ and B) with well-supported bootstrap values. Five and six OsSERK and OsSERL proteins were included in groups 

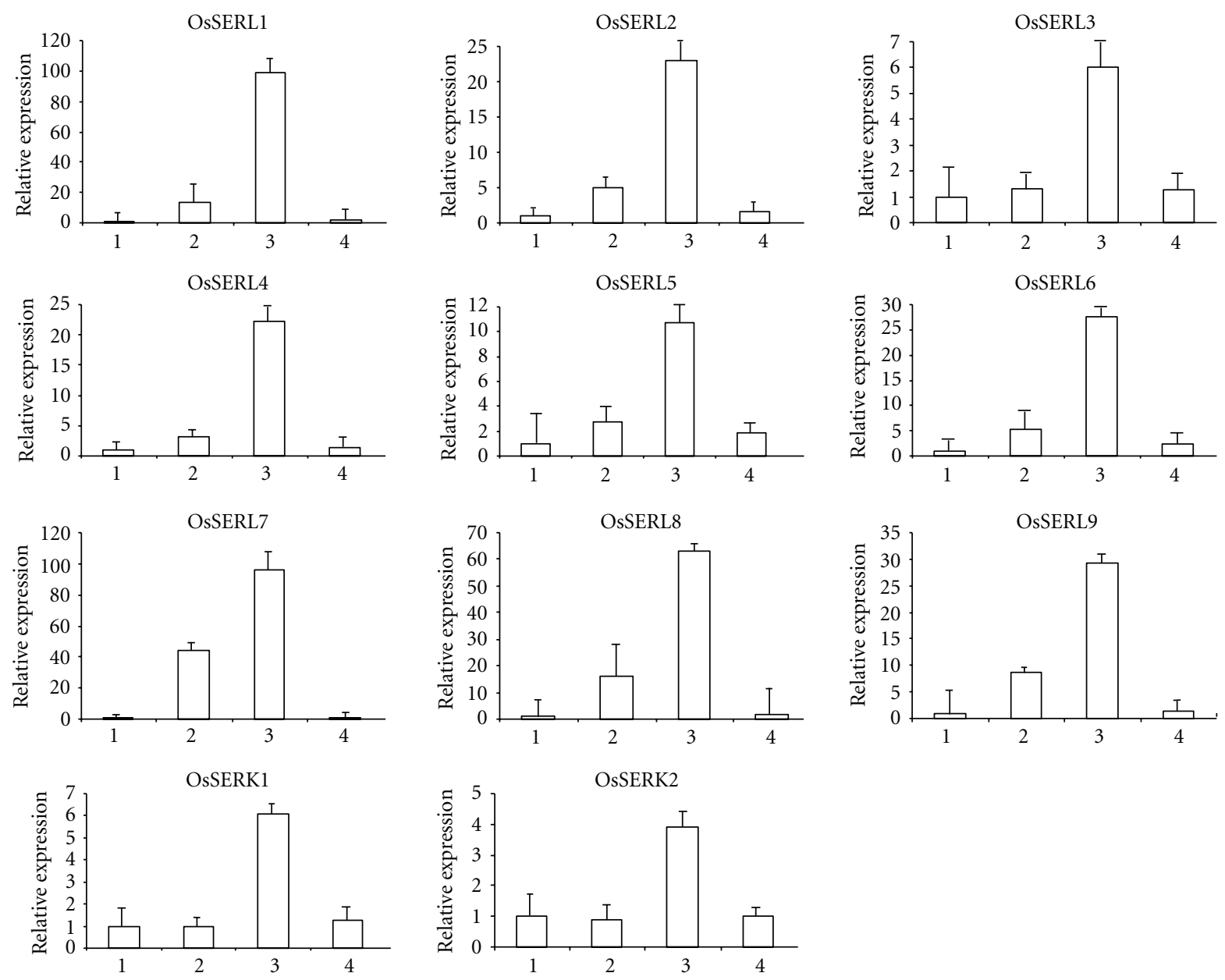

FIGURE 3: Real-time PCR expression profiles of individual SERK andSERL genes. (1) The relative mRNA levels of individual OsSERK and OsSERLgenes in pre-pollinated, (2) 1-2 days after pollination, (3) 5-10 days after pollination, and (4) 21 days after pollination from field grown rice seedlings. The relative mRNA levels of individual OsSERK and OsSERL genes normalized with respect to housekeeping gene Actin.

A and B, respectively. Groups A and B could be further sub-divided into two subgroups each (A1-A2 and B1-B2) with varying degree of bootstrap support. Most of the genes grouped together showed conserved gene structure, in terms of exon/intron organization and intron phasing. Eleven of the proteins formed four sister-pairs, two of them showing very strong bootstrap support (100\%). The phylogenetic tree with the SERK family in Arabidopsis, maize, sunflower, Poa pratensis, Gossypium hirsutum, and rice clustered the SERLs and the rest of the SERKs separately (see Supplementary Figure 2).

3.4. Differential Expression of OsSERK and OsSERL Genes. To determine the organ-specific expression of each OsSERK and OsSERL genes, real-time PCR was performed with total RNA isolated from different stages of rice seed development (days after pollination; DAP) (Figure 3) as well as dark-grown roots, etiolated shoots, green shoots, and roots (Figure 4). This analysis revealed that in all $11 \mathrm{cDNAs}$ of rice, expression is maximum at 5-10 DAP (Figure 3). In case of OsSERL proteins, there was a gradual increase in the expression level starting from prepollination to 5-10 DAP. At this stage, the embryonic phase and juvenile phase coexist, and enlargement and formation of embryonic organ continues [19]. OsSERL1 and OsSERL7 were the highest expressing followed by OsSERL8. OsSERK1, OsSERK2, and OsSERL3 depicted low activity as compared to the OsSERLs. At 21 DAP (developing seed), the expression decreased in all the genes examined (Figure 3). It has been reported that in Arabidopsis, AtSERK1 expressed in flowers at 3 DAP, containing developing seeds with embryos from stages 1 through 7 [20], although after fertilization AtSERK1 expression appeared to decrease rapidly and was detected in few cells of the developing seed [3]. Essentially similar has been the case for CiSERK1 transcripts which are highly abundant in fruits at $30 \mathrm{DAF}$ and $60 \mathrm{DAF}$ but not at $180 \mathrm{DAF}$ [21]. PpSERK1 expression was high during premeiosis and decreased during meiosis and postmeiosis [14]. It appears that, in general, SERKs and SERLs expression gradually decreases at the later stages, after fertilization. OsSERK1 and OsSERK2 show the least expression in various stages of developing seed as 

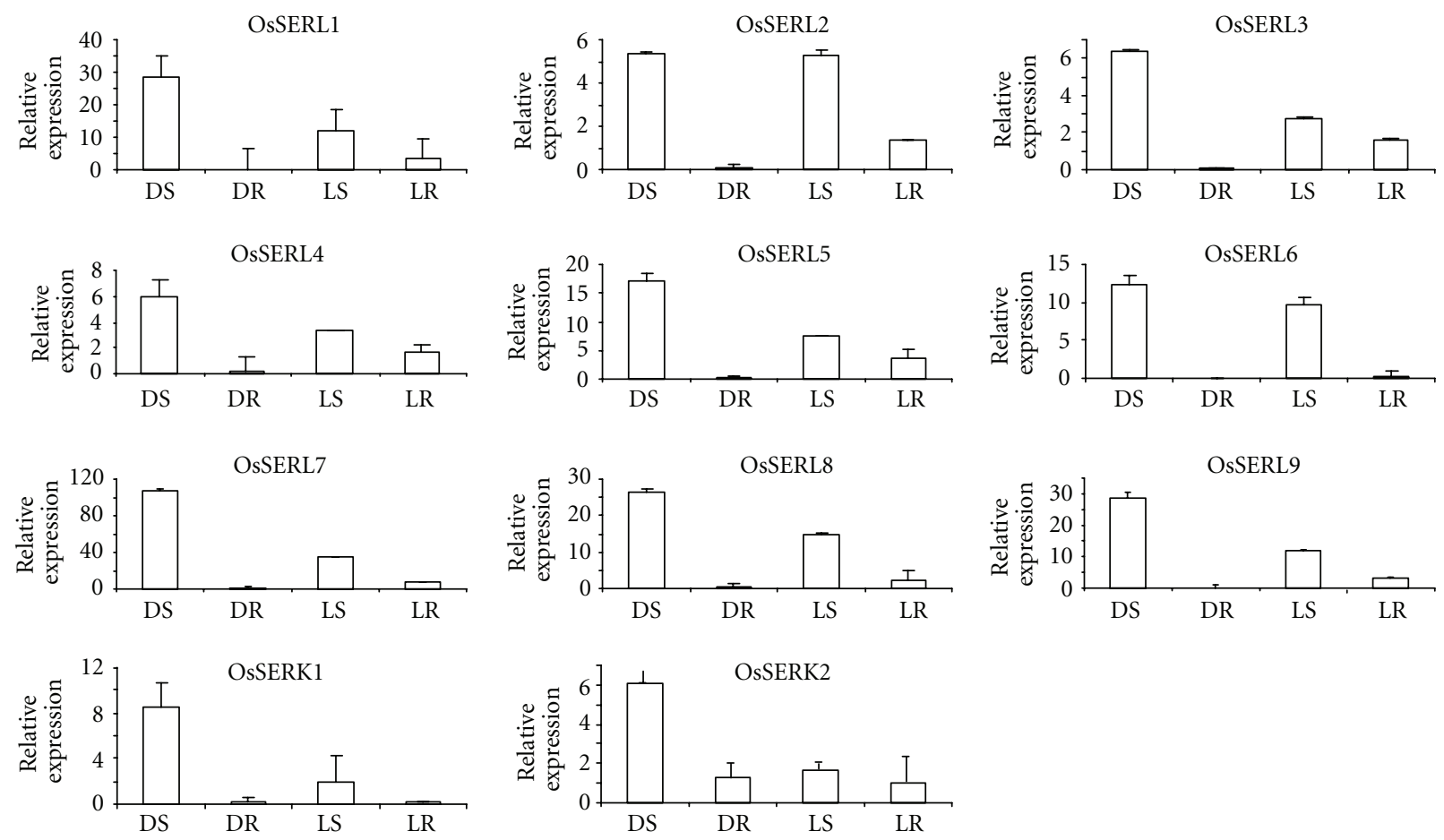

Figure 4: Real-time PCR expression profiles of individual SERK andSERL genes. The relative mRNA levels of individual OsSERK and OsSERL genes are normalized with respect to housekeeping gene Actin in different tissues (DS dark shoot, DR dark root, LS light shoot, and $L R$ light root).

compared to OsSERLs. Thus, it appears that the expression of OsSERK genes is probably confined or is more specific in somatic embryogenesis than in zygotic embryogenesis where they play a constitutive role as is evident from our data. Studies would be, however, required to check the expression pattern prior to fertilization in ovules as AtSERK1, ZmSERK1, ZmSERK2, and PpSERK2 show high expression in developing ovules $[2,3,14]$.

To check the expression in different organs of rice, total RNA from roots (dark and light grown), etiolated and green shoots, was isolated. OsSERK and OsSERL genes show a complexity of specific and overlapping expression patterns in various organs/tissues analyzed. Expression of OsSERKs and OsSERLs is higher in etiolated shoots (DS) than in green shoots (LS), except OsSERL2, which shows the same expression level in the two types of shoots, and all the OsSERLs show higher expression in light-grown roots (LR) than in dark-grown roots (DR); expression of the two OsSERKs is similar in light- and dark-grown roots: very low for OsSERK1 and higher for OsSERK2 (Figure 4). Exogenous auxin plays an important role in somatic embryo induction $[22,23]$. For OsSERL genes, the effect of auxin was not significant, except a distinct upregulation for OsSERL1,OsSERL2, and OsSERL7 (Figure 5(a)). Earlier studies have shown that auxin up-regulates MtSERK1 expression in both Medicago trunculataroot forming and embryogenic cultures [24]. The OsSERK and OsSERL genes showed complexity of specific and overlapping expression patterns in tissues or calli treated with the hormone auxin indicating that they might perform specific functions or act redundantly. Since SE can be categorized into inductive and maturation phase, it was imperative to investigate the SERK expression subsequent to auxin application. The difference in kinetics between individual OsSERK andOsSERL genes is likely due to a variety of factors, such as tissue-specific auxin perception, cell-type dependence and differential regulation of auxin concentration, or different modes of action of auxindependent transcriptional and posttrancriptional regulation. Among the two SERKs, OsSERK2 showed specific expression during the maturation phase (Figure 5(b)), and it appears to be more specific for somatic embryogenesis than OSSERK1. Earlier reports also support this observation [6]. With the exception of OsSERL3, all OsSERLs were downregulated, with OsSERL1 and OsSERL7 being most affected during the maturation phase (having a broader role in morphogenesis), and OsSERK2and OsSERL3 were upregulated (probably being specific to SE). The OsSERLs lacking the characteristic SPP motif, except OsSERL3, showed a decrease in transcript in the embryogenic calli (maturation phase), thus further supporting the fact that the presence of the SPP motif may be the essential factor for an SERK function. OsSERLs seem to have a broader role in morphogenesis in cultured tissue rather than being specific to somatic embryogenesis. Essentially similar observations have been made for TcSERK in cacao [25] and for MtSERK in Medicago trunculata [24]. In sunflower too, SERK transcripts accumulate early after the beginning of the culture in the morphogenetic zone of immature zygotic embryos (IZEs) of sunflower, 


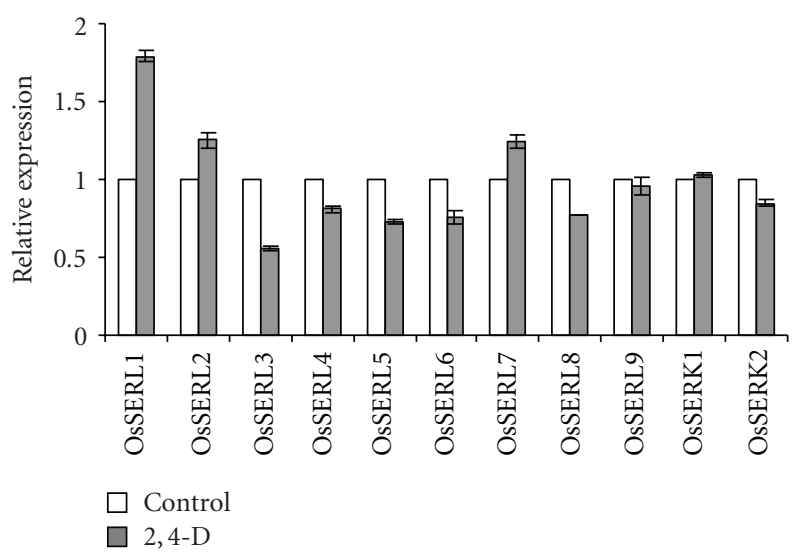

(a)

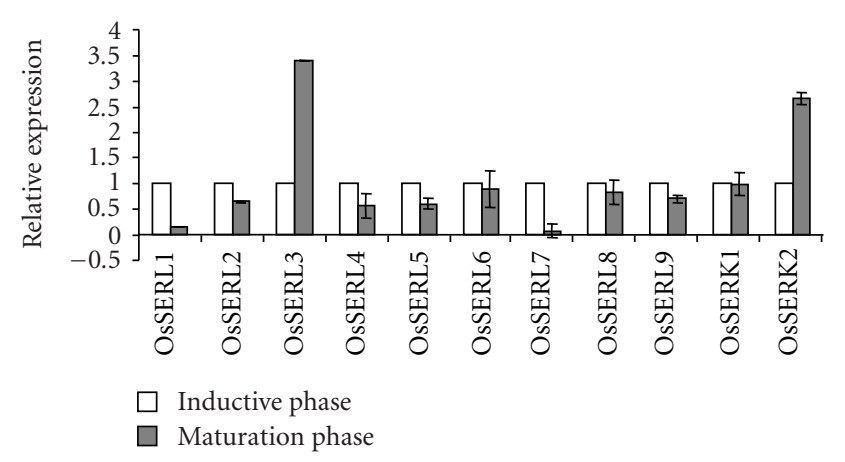

(b)

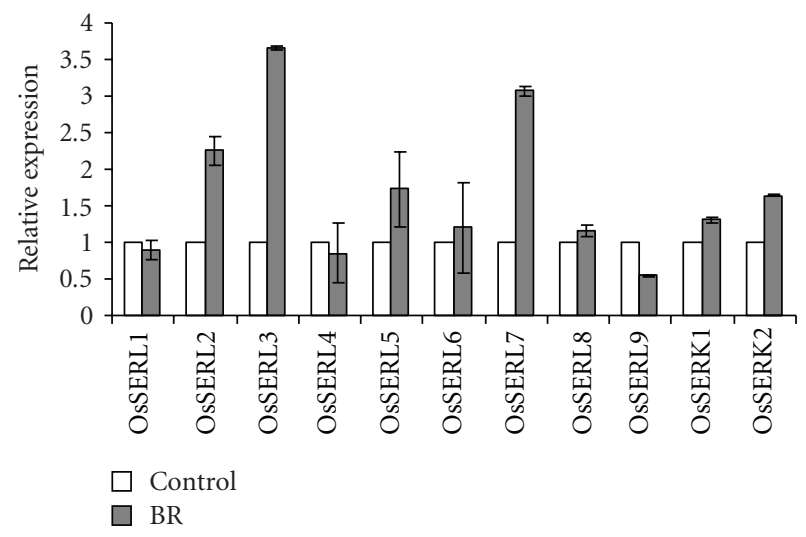

(c)

FIGURE 5: The relative mRNA levels of the SERK/SERL genes in (a) 3 -day-old etiolated seedlings in either water or $30 \mu \mathrm{M} 2,4-\mathrm{D}$, (b) embryogenic calli during induction and maturation phases, and (c) 3-day-old etiolated rice coleoptiles treated with $100 \mathrm{nM}$ BR.

whatever the induction conditions used, that is, organogenic, embryogenic, or highly embryogenic conditions [13].

In plants, systemic signaling molecules and hormones play key roles in establishing the developmental program and are also intimately involved in shaping plant growth and development. Studies have demonstrated a link between brassinosteroids and auxin indicating that some pathways are under dual control [26-28], and some signaling pathways converge at the level of the transcriptional regulation of common target genes $[28,29]$. It has been proposed that BR-induced effects are mediated via auxin or enhancing sensitivity to auxin $[26,30,31]$ and several researchers have provided insights into auxin and BR interactions $[32,33]$. Thus, to check the inducibility of these OsSERK and OsSERL genes by exogenous $\mathrm{BR}$, their expression was examined by real-time PCR, and eight of these genes were found to be induced in coleoptiles treated with BR (Figure 5(c)); maximum induction was recorded in OsSERL3 and OsSERL7. Also, in the last few years, studies have revealed the interaction between SERKs and other proteins like brassinosteroid insensitive (BRI) [34, 35]. Besides BRI receptor and its coreceptor, the AtSERK3 or BRI-associated kinase 1 protein, the Arabidopsis somatic embryogenesis receptor-like kinase protein complex has been shown to include kinase associated protein phosphatase, CDC48A, 143-3v, MADS box transcription factor AGAMOUS-LIKE15, and an uncharacterized zinc finger protein, a member of the CONSTANS family [35]. Further studies would be, however, required to unravel the role of this protein complex and the interactive effect of brassinosteroids and auxins in regulating somatic embryogenesis.

\section{Conclusions}

OsSERLs seem to have a broader role in morphogenesis rather than being specific to somatic embryogenesis. Functional validation of these somatic embryogenesis receptorlike kinase genes may help in elucidating their precise functions in regulating various facets of plant development.

\section{Acknowledgments}

The first author acknowledges the award of Senior Research Fellowship from the University of Grants Commission, New Delhi. This research work was financially supported by the Department of Biotechnology, Government of India, and the University Grants Commission, New Delhi. The authors gratefully acknowledge the KOME, TAIR, TIGR, and the IRGSP database resources for availability of detailed sequence information on rice.

\section{References}

[1] S.-H. Shiu and A. B. Bleecker, "Receptor-like kinases from Arabidopsis form a monophyletic gene family related to animal receptor kinases," Proceedings of the National Academy of Sciences of the United States of America, vol. 98, no. 19, pp. 10763-10768, 2001.

[2] S. Baudino, S. Hansen, R. Brettschneider, et al., "Molecular characterisation of two novel maize LRR receptor-like kinases, which belong to the SERK gene family," Planta, vol. 213, no. 1, pp. 1-10, 2001.

[3] V. Hecht, J.-P. Vielle-Calzada, M. V. Hartog, et al., "The Arabidopsis somatic embryogenesis receptor kinase 1 gene is expressed in developing ovules and embryos and enhances embryogenic competence in cultures," Plant Physiology, vol. 127, no. 3, pp. 803-816, 2001.

[4] S. Vij, V. Gupta, D. Kumar, et al., "Decoding the rice genome," BioEssays, vol. 28, no. 4, pp. 421-432, 2006. 
[5] L. G. Clark, W. Zhang, and J. F. Wendel, "A phylogeny of the grass family (Poaceae) based on ndhF sequence data," Systematic Botany, vol. 20, no. 4, pp. 436-460, 1995.

[6] H. Hu, L. Xiong, and Y. Yang, "Rice SERK1 gene positively regulates somatic embryogenesis of cultured cell and host defense response against fungal infection," Planta, vol. 222, no. 1, pp. 107-117, 2005.

[7] Y. Ito, K. Takaya, and N. Kurata, "Expression of SERK family receptor-like protein kinase genes in rice," Biochimica et Biophysica Acta, vol. 1730, no. 3, pp. 253-258, 2005.

[8] D. Song, G. Li, F. Song, and Z. Zheng, "Molecular characterization and expression analysis of OsBISERK1, a gene encoding a leucine-rich repeat receptor-like kinase, during disease resistance responses in rice," Molecular Biology Reports, vol. 35, no. 2, pp. 275-283, 2008.

[9] S. F. Altschul, T. L. Madden, A. A. Schäffer, et al., "Gapped BLAST and PSI-BLAST: a new generation of protein database search programs," Nucleic Acids Research, vol. 25, no. 17, pp. 3389-3402, 1997.

[10] J. D. Thompson, T. J. Gibson, F. Plewniak, F. Jeanmougin, and D. G. Higgins, "The CLUSTAL X windows interface: flexible strategies for multiple sequence alignment aided by quality analysis tools," Nucleic Acids Research, vol. 25, no. 24, pp. 4876-4882, 1997.

[11] Y. Hiei, S. Ohta, T. Komari, and T. Kumashiro, "Efficient transformation of rice (Oryza sativa L.) mediated by Agrobacterium and sequence analysis of the boundaries of the TDNA," Plant Journal, vol. 6, no. 2, pp. 271-282, 1994.

[12] B. Singla, A. Chugh, J. P. Khurana, and P. Khurana, "An early auxin-responsive Aux/IAA gene from wheat (Triticum aestivum) is induced by epibrassinolide and differentially regulated by light and calcium," Journal of Experimental Botany, vol. 57, no. 15, pp. 4059-4070, 2006.

[13] C. Thomas, D. Meyer, C. Himber, and A. Steinmetz, "Spatial expression of a sunflower SERK gene during induction of somatic embryogenesis and shoot organogenesis," Plant Physiology and Biochemistry, vol. 42, no. 1, pp. 35-42, 2004.

[14] E. Albertini, G. Marconi, L. Reale, G. Barcaccia, A. Porceddu, F. Ferranti, and M. Falcinelli, "SERK and APOSTART. Candidate genes for apomixis in Poa pratensis," Plant Physiology, vol. 138, no. 4, pp. 2185-2199, 2005.

[15] B. Singla, J. P. Khurana, and P. Khurana, "Characterization of three somatic embryogenesis receptor kinase genes from wheat, Triticum aestivum," Plant Cell Reports, vol. 27, no. 5, pp. 833-843, 2008.

[16] D. M. Braun and J. C. Walker, "Plant transmembrane receptors: new pieces in the signaling puzzle," Trends in Biochemical Sciences, vol. 21, no. 2, pp. 70-73, 1996.

[17] W. J. Fantl, D. E. Johnson, and L. T. Williams, "Signalling by receptor tyrosine kinases," Annual Review of Biochemistry, vol. 62, pp. 453-481, 1993.

[18] K. Shah, T. W. J. Gadella Jr., H. van Erp, V. Hecht, and S. C. de Vries, "Subcellular localization and oligomerization of the Arabidopsis thaliana somatic embryogenesis receptor kinase 1 protein," Journal of Molecular Biology, vol. 309, no. 3, pp. 641$655,2001$.

[19] J.-I. Itoh, K.-I. Nonomura, K. Ikeda, et al., "Rice plant development: from zygote to spikelet," Plant and Cell Physiology, vol. 46, no. 1, pp. 23-47, 2005.

[20] G. Jurgens and U. Mayer, "Arabidopsis," in Embryos. Color Atlas of Development, J. B. L. Bard, Ed., pp. 7-21, Wolf Publishers, London, UK, 1994.

[21] T. Shimada, T. Hirabayashi, T. Endo, H. Fujii, M. Kita, and M. Omura, "Isolation and characterization of the somatic embryogenesis receptor-like kinase gene homologue (CitSERK1) from Citrus unshiu Marc," Scientia Horticulturae, vol. 103, no. 2, pp. 233-238, 2005.

[22] A. Feher, T. P. Pasternak, and D. Dudits, "Transition of somatic plant cells to an embryogenic state," Plant Cell, Tissue and Organ Culture, vol. 74, no. 3, pp. 201-228, 2003.

[23] M. Quint and W. M. Gray, "Auxin signaling," Current Opinion in Plant Biology, vol. 9, no. 5, pp. 448-453, 2006.

[24] K. E. Nolan, R. R. Irwanto, and R. J. Rose, "Auxin upregulates MtSERK1 expression in both Medicago truncatula root-forming and embryogenic cultures," Plant Physiology, vol. 133, no. 1, pp. 218-230, 2003.

[25] M. de Oliveira Santos, E. Romano, K. S. C. Yotoko, M. L. P. Tinoco, B. B. A. Dias, and F. J. L. Aragão, "Characterisation of the cacao somatic embryogenesis receptor-like kinase (SERK) gene expressed during somatic embryogenesis," Plant Science, vol. 168, no. 3, pp. 723-729, 2005.

[26] N. B. Mandava, "Plant growth-promoting brassinosteroids," Annual Review of Plant Physiology and Plant Molecular Biology, vol. 39, pp. 23-52, 1988.

[27] F. Bao, J. Shen, S. R. Brady, G. K. Muday, T. Asami, and Z. Yang, "Brassinosteroids interact with auxin to promote lateral root development in Arabidopsis," Plant Physiology, vol. 134, no. 4, pp. 1624-1631, 2004.

[28] A. Nakamura, N. Nakajima, H. Goda, et al., "Arabidopsis Aux/IAA genes are involved in brassinosteroid-mediated growth responses in a manner dependent on organ type," Plant Journal, vol. 45, no. 2, pp. 193-205, 2006.

[29] B. Singla, A. K. Tyagi, J. P. Khurana, and P. Khurana, "Analysis of expression profile of selected genes expressed during auxininduced somatic embryogenesis in leaf base system of wheat (Triticum aestivum) and their possible interactions," Plant Molecular Biology, vol. 65, no. 5, pp. 677-692, 2007.

[30] A. Nakamura, K. Higuchi, H. Goda, et al., "Brassinolide induces IAA5, IAA19, and DR5, a synthetic auxin response element in Arabidopsis, implying a cross talk point of brassinosteroid and auxin signaling," Plant Physiology, vol. 133, no. 4, pp. 1843-1853, 2003.

[31] A. Sakurai, T. Yokota, and S. D. Clouse, "Physiological actions of brassinosteroids," in Brassinosteroids: Steroidal Plant Hormones, pp. 137-161, Springer, Tokyo, Japan, 1999.

[32] K. J. Halliday, "Plant hormones: the interplay of brassinosteroids and auxin," Current Biology, vol. 14, no. 23, pp. R1008R1010, 2004.

[33] K. Mockaitis and M. Estelle, "Integrating transcriptional controls for plant cell expansion," Genome Biology, vol. 5, no. 11, article 245, 2004.

[34] E. Russinova, J.-W. Borst, M. Kwaaitaal, et al., "Heterodimerization and endocytosis of Arabidopsis brassinosteroid receptors BRI1 and AtSERK3 (BAK1)," Plant Cell, vol. 16, no. 12, pp. 3216-3229, 2004.

[35] R. Karlova, S. Boeren, E. Russinova, J. Aker, J. Vervoort, and S. de Vries, "The Arabidopsis somatic embryogenesis receptorlike kinase1 protein complex includes brassinosteroidinsensitive 1," Plant Cell, vol. 18, no. 3, pp. 626-638, 2006. 

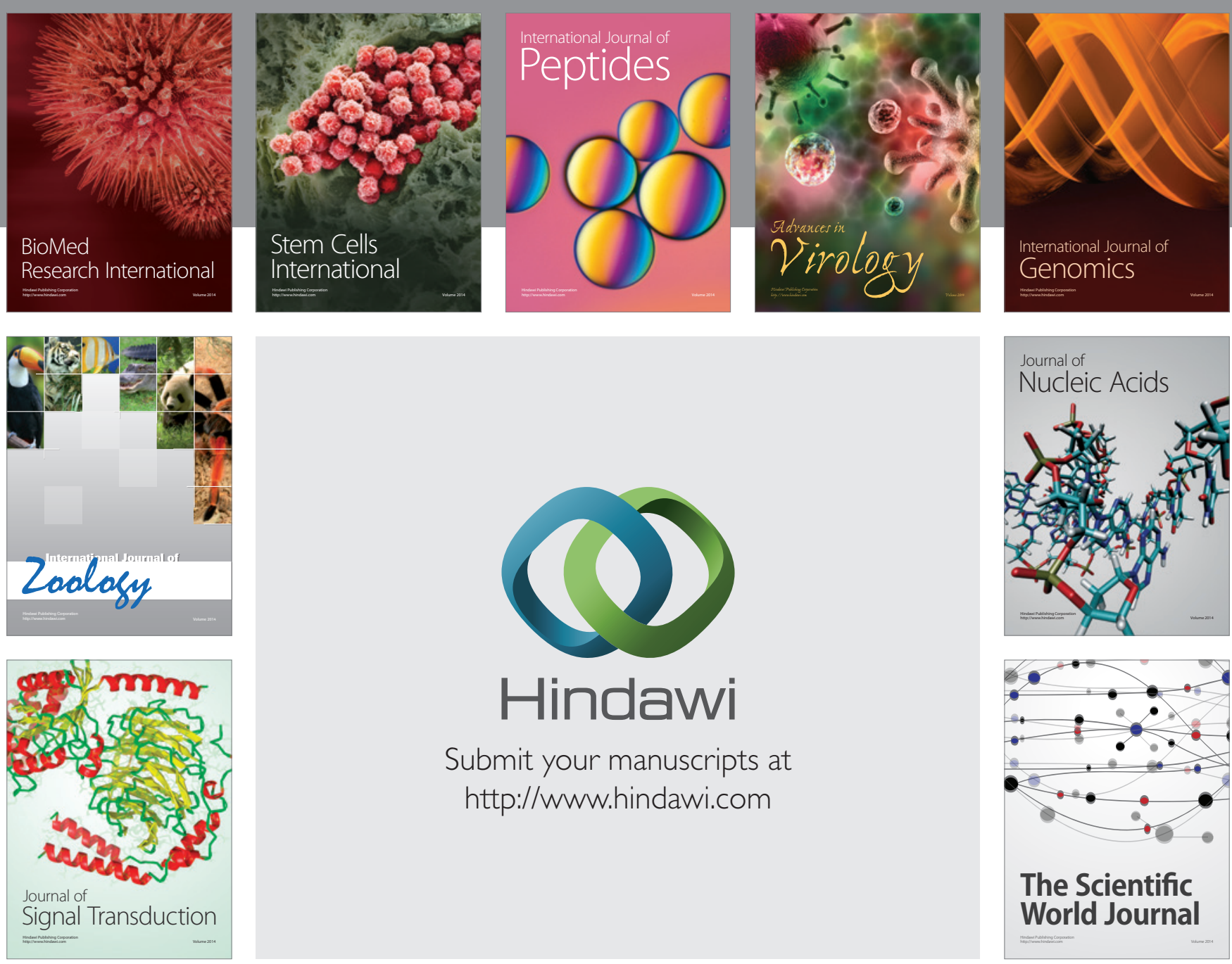

Submit your manuscripts at

http://www.hindawi.com
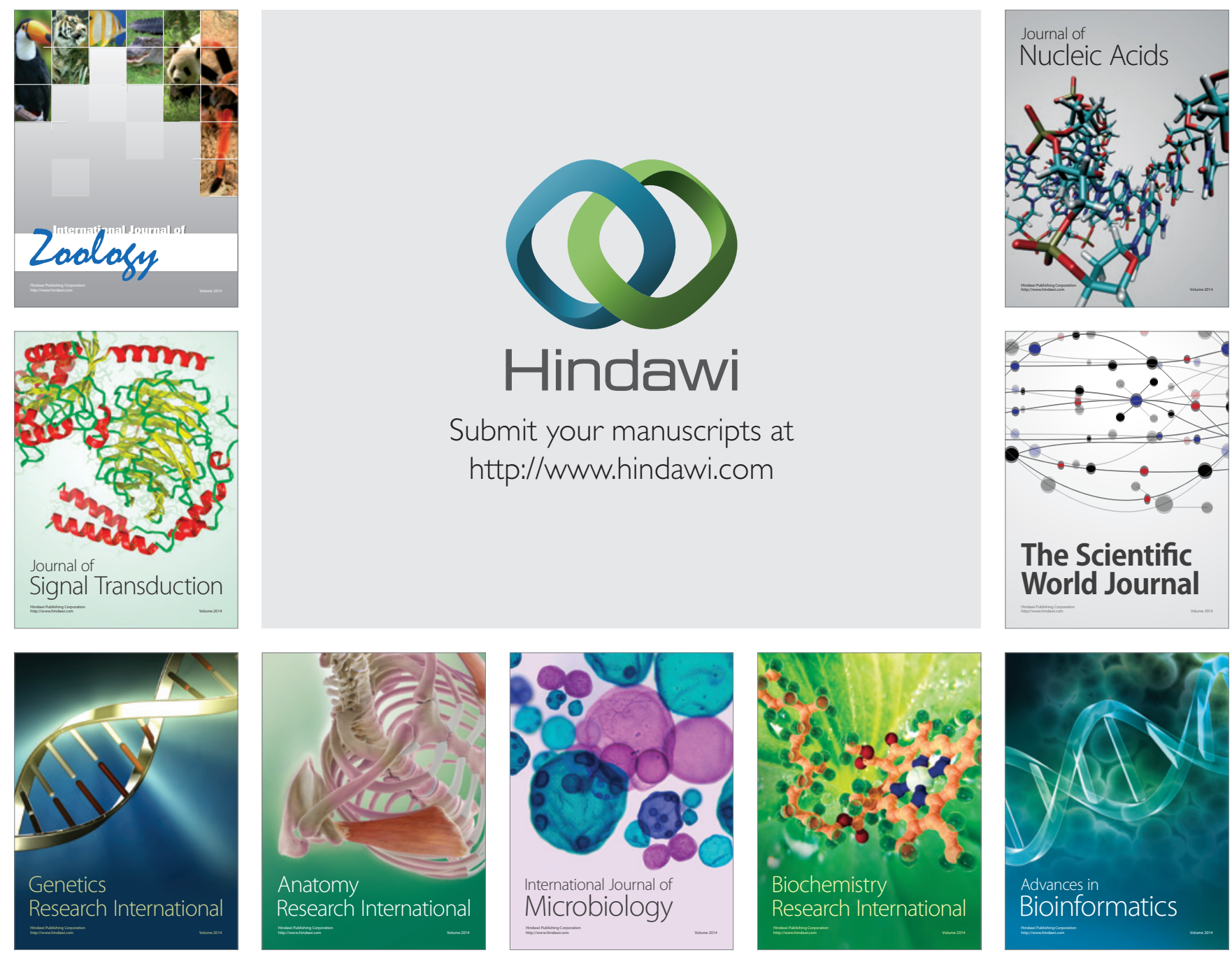

The Scientific World Journal
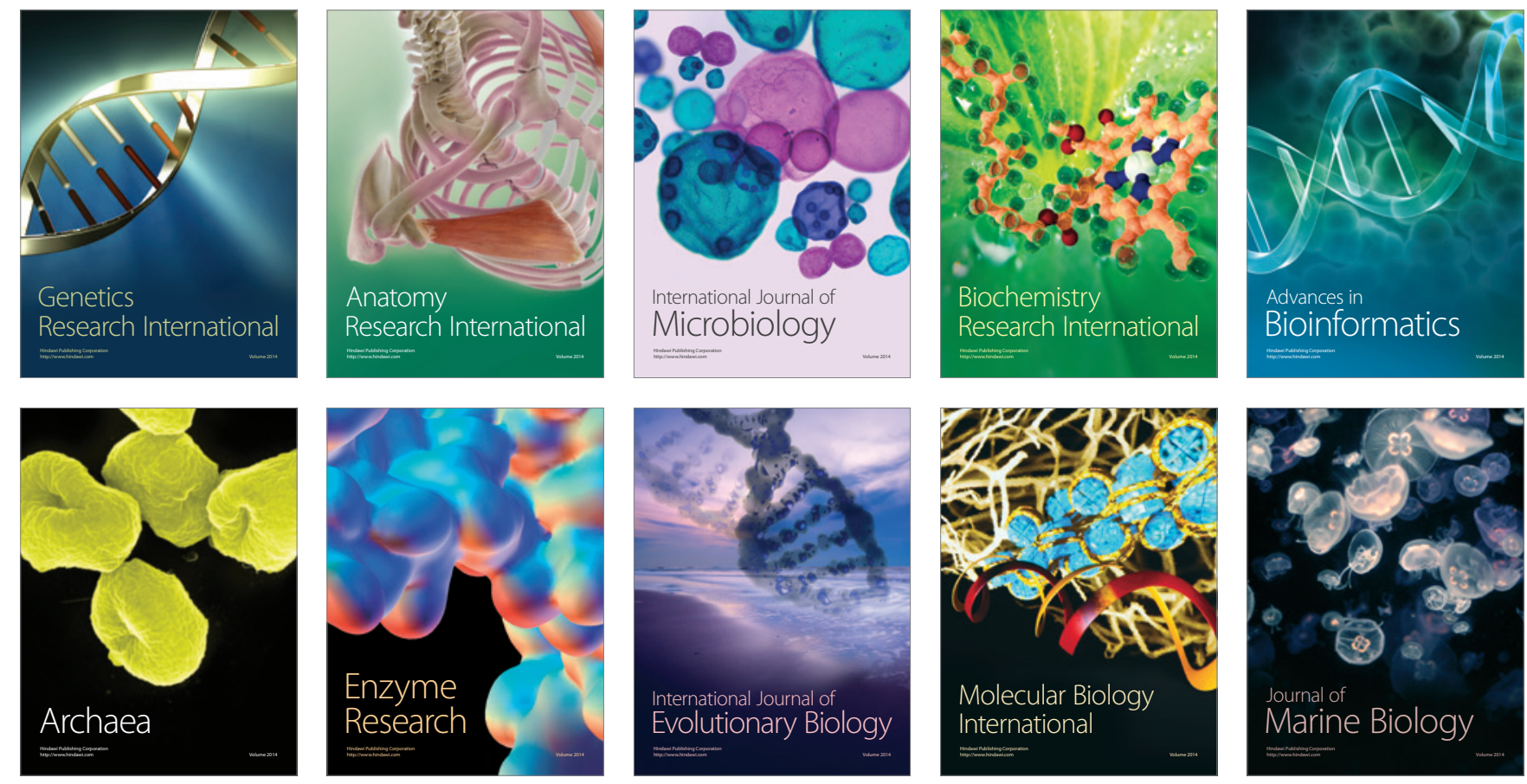